\title{
Leadership participatif et identification organisationnelle : rôle médiateur de la responsabilité sociale des entreprises perçue par les employés et la fierté d'être membre dans les petites et moyennes entreprises béninoises
}

\author{
Zinsou Daniel NAKOU ${ }^{1}$, Serge Francis SIMEN NANA ${ }^{2}$ \\ ${ }^{1}$ Ecole Supérieure Polytechnique, Université Cheikh Anta Diop, Dakar, Sénégal \\ ${ }^{2}$ Ecole Supérieure Polytechnique, Université Cheikh Anta Diop, Dakar, Sénégal
}

\begin{abstract}
Résumé : Cet article étudie l'influence du Leadership Participatif (LP) sur l'Identité Organisationnelle (IO) au moyen de la Responsabilité Sociale des Entreprises (RSE) perçue par les employés et de la Fierté d'Etre Membre (FEM) sous l'angle de la théorie du traitement de l'information sociale dans les PME béninoises. Pour atteindre cet objectif, nous avions utilisé une méthode quantitative basée sur un questionnaire soumis à 740 employés de 17 Petites et Moyennes Entreprises (PME) réparties dans trois (03) villes au Bénin (5 à Cotonou, 7 à Porto-Novo et 5 à Parakou) et dont les données ont été présentées et traitées avec le logiciel SPSS 21.0. Nos résultats montrent que le LP conduit à des perceptions internes positives des employés en matière de RSE, ce qui fait appel à la gestion de l'information personnelle qui, à son tour, debouche sur une IO. En outre, les perceptions internes de la RSE ont servi de médiaton à la relation entre le LP et la FEM ; puis la FEM, de médiation entre les perceptions internes de la RSE et l'IO dans les PME sélectionnées. Les implications de ces résultats sont également discutées.
\end{abstract}

Mots-clés : Théorie du traitement de l'information sociale, Responsabilité sociale des entreprises, Leadership participatif, Identification organisationnelle, Fierté d'être membre, Petites et moyennes entreprises.

\section{INTRODUCTION}

Très peu de littérature explique la participation des petites et moyennes entreprises (PME) à la responsabilité sociale des entreprises (RSE) (Inyang, 2013). Les PME, entreprises de moins de 250 salariés (Hall et al., 2009), représentent plus de $90 \%$ des entreprises mondiales et générent près de $60 \%$ des emplois dans le monde (Jamali et al., 2009). Une plus grande attention s'avère impérative pour comprendre les distinctions potentielles et les contributions des PME en matière de RSE (Inyang, 2013). Pour combler ces lacunes dans la littérature, cet article se propose de tester le modèle élaboré dans les PME béninoises.

Autour du concept de la RSE, gravite un flou et une ambiguité entretenue par la pléthore de définitions et d'approches. Ce flou est entretenu par les enjeux théoriques, managériaux et idéologiques (Locket et al, 2006). Selon Allouche et al (2004, p85) cité par Yedder et Zedden (2009) «La RSE et les outils auxquels elle a donné lieu évoluent dans un cadre conceptuel où règnent la confusion et l'imprécision ». Toutefois, comme le suggère Pesqueux (2018, p1), la RSE concerne « la prise en compte des demandes des parties prenantes comme 
formalisation de la réponse à une demande sociale venant alors confondre responsabilité sociale, réceptivité et intégration des pratiques liées à cette notion aux logiques de gestion, expression de son «pouvoir social » en quelque sorte et comportant l'idée de « vouloir faire quelque chose » afin de donner un contenu social à l'exercice du volontarisme managérial voire de s'assurer quant au risque de réputation ». Ainsi, la RSE perçue des employés est définie comme le degré avec lequel ceux-ci voient si leur entreprise est impliquée ou non dans des pratiques de RSE (Choi et Yu, 2018). Puisque la manière dont les activités de RSE d'une organisation sont perçues par les employés est un facteur important dans la compréhension des effets de ces activités au niveau individuel (Shin et al, 2016). Certaines études ont été consacrées à la détermination des antécédents de perceptions de la RSE, tels que l'adéquation perçue des employés entre la culture d'entreprise conjuguée aux activités de RSE de l'entreprise et la performance spécifique de la RSE perçue dans le rôle du responsable (Lee et al., 2017). A titre d'exemple, des appels ont été lancés pour déterminer d'autres antécédents de la RSE perçus (Tian et al., 2015).

Cependant, la relation chef-suiveur est l'une des discussions les plus centrales dans le monde des affaires. Un noble leader est essentiel pour obtenir de bons résultats chez les employés et améliorer leurs aspects émotionnels de manière positive (Choudhary et al., 2013). Un nombre limité d'études ont exploré le rôle que le leadership joue dans l'identification organisationnelle des employés. La plupart de ces études ont principalement porté sur le leadership transformationnel et transactionnel (Epitropaki et Martin, 2015). Le leadership participatif est cependant relativement nouveau pour la perspective de la recherche sur le leadership (Martin, 2015). Ce style de leadership qui favorise la consultation et la discussion en rapport avec la direction (Amabile et al., 2004) s'est révélé être positivement associé aux résultats souhaités par les employés, tels que l'engagement organisationnel, la satisfaction au travail, le rendement au travail, le comportement de citoyen de l'organisation et l'innovation en équipe (Chen et al, 2016s), mais n'a pas encore été lié à l'identification organisationnelle (IO). Cette dernière est la vision de l'appartenance d'un membre à une organisation de telle sorte que son adhésion à cette organisation se transforme en une partie substantielle de la définition de soi de la personne (Ashforth et Mael, 1989).

De plus, malgré la curiosité accrue dans le lien entre leadership et RSE, peu de recherches étonnantes sont excitantes sur cette intersection (Strand, 2016). Quelques études ont lié le leadership à la RSE et ont principalement porté sur les styles de leadership éthique, transformationnel et transactionnel (Groves et La Rocca, 2015). Néanmoins, des études supplémentaires sur les styles de leadership et leur relation avec la RSE sont essentielles car le leadership reste un concept primordial qui manque d'investigation (Martin, 2015). Par conséquent, en examinant la relation entre leadership participatif et perceptions de la RSE en référence à la théorie du traitement de l'information sociale (Salancik et Pfeffer, 1978), cette étude est l'une des premières à proposer que le leadership participatif soit un antécédent de perceptions positives des employés en matière de RSE. Par ailleurs, plusieurs études ont exploré l'association de la RSE avec l'IO (Jacinto et Carvalho, 2019). D'ailleurs, les conclusions de la plupart des études indiquent que la RSE intensifie l'IO des employés (Jacinto et Carvalho, 2019). Dans cette perspective, De Roeck et al (2014) appellent les recherches futures à trouver des mécanismes potentiels dans la relation entre les perceptions de la RSE et l'IO. Ils recommandent également aux chercheurs d'étudier la fierté d'être membre (FEM) en termes de littérature sur la RSE. La FEM exprime à quel point les individus ressentent le respect de soi comme conséquence de leur appartenance à l'organisation (Jones, 2010). Aussi, des appels ont-ils été lancés pour étudier l'influence de la fierté sur l'IO (Ayse et al, 2015). Cette étude répond à ces demandes de recherche en testant les éventuels effets médiateurs de la gestion d'informations personnelles sur la relation entre les perceptions internes de la RSE et l'IO en référence à la théorie de l'identité sociale (Tajfel et Turner, 1979). Le modèle de médiation séquentiel de cette recherche testée propose que le leadership participatif incite à une perception positive de la RSE interne qui conduit à la FEM. 
Dans le cadre de cet article, nous nous interrogeons : Comment le leadership participatif affecte-t-il l'identification organisationnelle des employés en matière de RSE au moyen de la fierté d'appartenance dans l'affiliation de leurs perceptions de la RSE?

Avant de présenter les résultats de cette recherche, nous convoquons une synthèse de la littérature portant sur les concepts et théories pour ensuite mieux expliquer notre démarche méthodologique et enfin, nous présentons et discutons nos résultats et les implications managériales qui en découlent.

\section{REVUE DE LA LITTERATURE ET DEVELOPPEMENT DES HYPOTHESES}

\section{A. RSE dans les PME}

Bien qu'il soit difficile d'identifier un schéma global illustratif des facteurs expliquant le développement des pratiques de RSE dans les PME, par opposition aux grandes entreprises (Herrera et al., 2016), certaines caractéristiques influent cependant sur l'approche des PME en matière de RSE. La littérature sur le sujet a révélé certains facteurs favorisant la RSE dans les PME, tels que l'âge de la société (Santos et al., 2006), la taille de la société (Preuss et Perschke, 2010), l'emplacement de l'activité (COM, 2002a), le secteur d'activité (Herrera et al., 2016).

Selon Fassin (2008), les PME utilisent moins les instruments de RSE que les grandes entreprises, ont moins de stratégies formelles de RSE, sont moins susceptibles de déclarer une activité de RSE et disposent de moins de ressources pour investir dans des pratiques de RSE (Perrini et al, 2007). En général, il est plus difficile d'impliquer les PME dans la RSE par rapport aux grandes entreprises (Davies et Crane, 2010), en raison notamment de problèmes de manque de temps et de ressources (Spence et Rutherfoord, 2001). Les grandes entreprises sont souvent plus en mesure d'absorber des coûts fixes, en plus d'avoir un meilleur accès aux ressources (Kechiche et Soparnot, 2012).

Selon Kechiche et Soparnot (2012), il existe des facteurs tels que la taille de l'entreprise, la localisation et les caractéristiques individuelles liées à la personnalité du directeur de la PME, communément admis en tant qu'influenceur de la décision de mettre en œuvre une politique de RSE responsable. En effet, la taille d'une entreprise est un facteur organisationnel dans l'acceptation et la mise en œuvre de la RSE par les PME (Labelle et Saint Pierre, 2010). Certaines études suggèrent qu'il existe un lien positif entre la taille d'une entreprise et son engagement en matière de RSE (Lepoutre et Heene, 2006 ; Perrini et al., 2007). Les grandes entreprises sont plus sensibles aux questions de RSE que les petites ou micro-entreprises, en particulier sur le plan environnemental (Sharma, 2000).

Bien que la taille soit un facteur, il existe d'autres dynamiques internes et externes qui expliquent leurs traits de comportement (Jenkins, 2006). Le comportement des PME est souvent associé aux caractéristiques psychologiques de l'entrepreneur ou du partenaire dirigeant (Jenkins, 2006). En règle générale, la gestion et le contrôle des PME sont essentiellement assurés par l'entrepreneur ou l'associé directeur de l'entreprise, ce qui donne une légitimité aux décisions personnelles prises quant à l'utilisation des ressources de l'entreprise et permet une certaine autonomie dans l'approche de la RSE (Jenkins, 2006).

En ce qui concerne les interactions entre la société et les entreprises, les PME travaillent principalement pour les communautés dans lesquelles elles sont insérées, car elles constituent par définition une seule unité opérationnelle fonctionnant avec une main-d'œuvre locale (Jenkins, 2006). Certains auteurs estiment que la cohésion territoriale et le point de vue local de l'entreprise peuvent influer sur la mise en œuvre des initiatives de RSE. La plupart des PME ont de solides attachements locaux et sont généralement identifiées à la région où 
elles sont implantées. En outre, la zone locale offre aux entreprises le bénéfice de nombreuses ressources, telles que l'infrastructure, la force de travail, les matières premières, et prévoit en retour des avantages économiques et communautaires par le biais de la réciprocité (Kechiche et Soparnot, 2012). Leurs homologues Lepoutre et Heene (2006) estiment que les PME sont plus sensibles aux pratiques des acteurs internes (employés, clients et fournisseurs), qui sont généralement les trois acteurs les plus influents pour les PME (Perrini et al., 2009).

\section{B. Traitement de l'information sociale}

Le modèle du traitement de l'information sociale concernant le comportement d'individu a marqué de sérieuses avancées dans la compréhension de leur ajustement social. Il permet aussi bien de faire le lien entre cognition sociale et adaptation sociale que de dégager des patterns de traitement de l'information sociale pouvant expliquer la manifestation d'un comportement relevant d'une mauvaise adaptation sociale comme l'agressivité. D'abord présenté par Dodge en 1986, il a ensuite été reformulé par Crick et Dodge en 1994 et par Lemerise et Arsenio en 2000.

Dans sa première version, Dodge (1986) part du principe que l'individu dispose de « capacités biologiques » lui permettant de gérer des interactions avec autrui et ce, grâce à des indices sociaux déjà présents en mémoire (ajustement social). Puis, il propose que l'individu passe par quatre (04) étapes mentales organisées de manière séquentielle avant de produire un comportement social (Figure I).

\section{FIGURE I : MODÈLE DU TRAITEMENT DE L'INFORMATION SOCIALE}

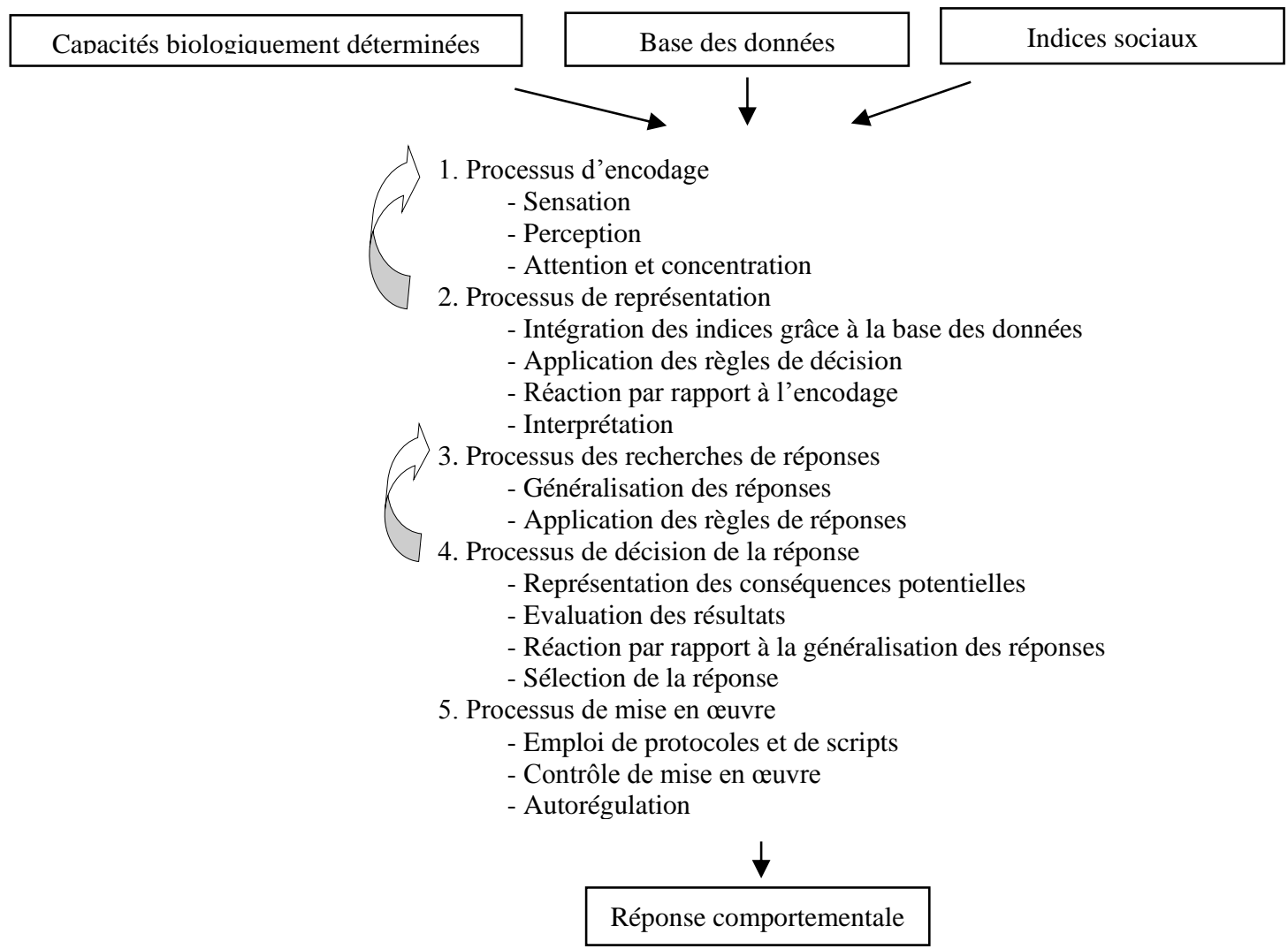

Source : Adapté de Dodge (1986) dans Huré (2014)

A travers cette figure, aux étapes 1 et 2, il fait l'hypothèse que les employés encodent les indices particuliers liés à la sensation et à la perception de la situation et construisent une inférence à propos de l'intention du pair 
avec lequel il interagit. Ces deux étapes interagissent très fortement l'une avec l'autre puisqu'en fonction de l'interprétation d'un indice, l'individu peut modifier l'encodage des indices suivants.

Aux étapes 3 et 4, il propose que l'individu accède aux possibles réponses aux situations qui sont stockées en mémoire à long terme, évalue ses solutions et sélectionne la plus pertinente à ses yeux. Il procède également à l'évaluation de la réussite et de l'efficacité de la réponse pour finir par sélectionner celle qui lui semble la plus adaptée.

A l'étape 5, l'individu produit la réponse selon les étapes précédemment réalisées. Puisque le modèle de Dodge (1986) décrit de manière spécifique la façon dont l'individu traite l'information sociale, il a servi de guide à de nombreuses recherches concernant les enfants présentant des difficultés d'ajustement social, et a également fait l'objet d'une réorganisation par Crick et Dodge (1994).

\section{Leadership participatif et implication des employés}

Les recherches existantes sur le leadership dans le contexte de la RSE sont plutôt fortement influencées par une seule dimension : A quel point les dirigeants des entreprises sont suffisamment informés sur la politique de RSE et dans quelle mesure ils l'appuient? Mais, apposer une signature au bas des directives éthiques pour le personnel ne signifie pas que le dirigeant exerce un leadership éthique et qu'il dirige ses employés vers une pensée et une action responsables, en donnant un sens à la vie organisationnelle et en établissant des relations avec les parties prenantes. Pour améliorer le contexte éthique du leadership, nous pouvons prendre en compte un modèle de participation ou de co-leadership.

Le leadership participatif est défini comme un leadership impliquant les employés à tous les niveaux de la hiérarchie dans la prise de décision (Spreitzer, 2007). Les leaders participatifs impliquent leurs subordonnés dans la prise et la mise en œuvre des décisions. Ils recherchent les commentaires de leurs subordonnés sur les décisions importantes et valorisent les points de vue des uns et des autres. Les systèmes participatifs permettent aux employés d'utiliser leurs voix pour influencer la stratégie interne et externe, protéger la réactivité de l'organisation et responsabiliser les gestionnaires. Lorsque les employés participent, ils considèrent le système dans son ensemble comme plus équitable, ce qui réduit les chances de traiter la responsabilité des entreprises comme une simple astuce de relations publiques. Grâce à cette responsabilisation, les employés font partie des moyens plus transparents d'interactions avec leurs pairs et les autres parties prenantes. Ils peuvent voir les avantages réels de ces pratiques participatives pour eux-mêmes au sens moral, et pour leur entreprise dans un sens plus opérationnel. Cela signifie également que la gestion des relations humaines (GRH), traditionnellement basée sur une approche instrumentale, devrait progressivement évoluer vers la gestion de la valeur humaine $(\mathrm{GVH})$ et qu'au lieu de faire respecter le comportement souhaité des employés, elle devrait créer des réseaux entre employés liés entre eux par des valeurs partagées, permettant l'organisation de fonctionner comme un système ouvert (Schoemaker et al., 2006). Selon ces auteurs, il est de plus en plus nécessaire de développer consciemment une compréhension approfondie des différents facteurs nécessaires au processus de construction des communautés de travail afin de parvenir aux résultats tangibles et intangibles. Ce passage des ressources aux valeurs devrait s'appuyer sur de nouveaux instruments qui vont au-delà des instruments traditionnels de gestion des ressources humaines, avec un engagement actif dans la prise de conscience, l'interconnexion et la signification du travail de manière responsable. Le défi consiste à intégrer une attitude axée sur les valeurs au niveau des individus et contraint à aller au-delà du type classique de leadership hiérarchique enraciné dans l'imposition et l'application de la loi dans le sens du modèle de leadership plus participatif. Il ne suffit pas de discuter du rôle changeant de l'entreprise en tant qu'acteur de la société dans l'environnement extérieur, mais aussi de souligner le lien étroit qui existe entre RSE et GRH. Dans différentes enquêtes sur les attentes du public 
concernant l'entreprise responsable, le principal facteur d'évaluation de la RSE repose sur la perception selon laquelle une entreprise traite ses employés équitablement (Googins et al., 2007).

De plus en plus d'ouvrages sur la participation et l'autonomisation des employés relatent que, plus les divers aspects d'une organisation sont participatifs, plus les chances de voir son efficacité sont conséquentes (Sumers et Hyman, 2005). La responsabilisation des employés est le facteur crucial de l'intégration réussie de la stratégie de RSE et doit être basée sur les valeurs et les visions partagées par tous les membres de l'organisation. Certains auteurs (Bainbridge, 1996) examinant différentes formes de programmes d'implication des employés prennent en compte deux catégories de base, allant du moins intrusif au plus intrusif : la participation opérationnelle et la participation stratégique.

\section{Leadership participatif et perceptions de la RSE}

Plusieurs chercheurs soutiennent que l'approche de leadership la plus humaniste est le style participatif (Amabile et al, 2004). Sous un style de leadership participatif, le dirigeant rencontre les employés pour discuter des problèmes de l'entreprise avant de prendre certaines décisions (Chen et Tjosvold, 2006). Un leader participatif, au lieu de prendre des décisions autocratiques, encourage modestement la participation des employés à la prise de décision et à la résolution de problèmes (Miao et al, 2013). Un certain nombre de spécialistes ont depuis longtemps affirmé que la participation des employés à certaines décisions méritait une attention empirique, car leur participation devait être d'une grande valeur pour l'entreprise et ses employés (Chen et Tjosvold, 2006). Bien que le leadership participatif puisse être considéré comme l'un des types de prise de décision les plus lents, lorsqu'une décision est prise collectivement, une harmonie est créée au sein de l'entreprise et le moral des employés ainsi que le soutien qu'ils ressentent au sein de l'organisation sont améliorés (Muindi, 2011). Somech (2005) pense que le processus de communication ouverte inauguré dans le leadership participatif peut aider à réduire les obstacles rencontrés par les membres de l'organisation. Il règle les conflits en construisant un travail d'équipe cohérent, car il améliore la compréhension de l'entreprise et l'acceptation des décisions prises (Sorenson, 2000). Bryman (2013) affirme que le leadership participatif est censé créer des employés plus heureux et plus productifs, car ils ont le sentiment d'avoir leur mot à dire et, par conséquent, un certain contrôle sur certains événements de l'entreprise. Quant à Strand (2011), le rôle que le leadership participatif peut jouer dans la RSE est une promesse de recherche. La RSE va au-delà de la recherche de l'intérêt personnel ou du profit et du respect de la loi (Aguilera et al, 2007) dans la mesure où elle se concentre sur la réalisation d'un bien social (McWilliams et Siegel, 2001). Elle a été considérée comme l'activité fondamentale dans la formation de relations entre parties prenantes (Bhattacharya et al, 2009). Elle peut être séparée en deux catégories. La première catégorie est la RSE externe qui comprend des activités liées à une cause distincte de l'entreprise, dirigées vers des parties prenantes externes. Cela peut prendre la forme d'une implication dans un projet social, comme parrainer un événement ou faire un don à un organisme de charité. La deuxième catégorie est la RSE interne, qui comprend les activités liées aux employés et au mode de fonctionnement interne de l'entreprise (Basil et Erlandson, 2008). Ces activités sont directement associées à l'environnement de travail psychologique et physique (Turker, 2009) et concernent le bien-être, la santé et la sécurité des employés (Vives, 2006). Cela peut prendre la forme de politiques de sécurité ou d'un code d'éthique bien établi (Basil et Erlandson, 2008). Ces mesures sont également exprimées en tant qu'activités préoccupantes pour la formation des employés, l'égalité des chances et l'équilibre travail-vie personnelle (Vives, 2006). Les exemples incluent des politiques de travail non discriminatoires et une déclaration claire sur les heures de travail (Welford, 2005).

Le concept de la RSE étant de nature interdisciplinaire (Lockett et al, 2006), l'aspect ressources humaines est un thème clé de la RSE (Berber et al, 2014). Sous cet aspect, les principales caractéristiques de la RSE interne comprennent les pratiques de gestion des ressources humaines liées à la composition du personnel, à la formation, à l'égalité de traitement, aux horaires de travail, à une répartition équitable des salaires, à l'éducation, 
aux avantages sociaux, à la santé et à la sécurité. La gestion des ressources humaines est une composante essentielle de la RSE interne, car les entreprises doivent exercer leurs activités de manière responsable dans toutes leurs fonctions de ressources humaines, du recrutement à la rétention. Pour cette raison, il existe un chevauchement entre les pratiques de GRH et les activités de RSE internes (Kochar et Bisht, 2014). Jamali et al (2015) conviennent que, pour atteindre ses objectifs, la RSE doit être abordée comme une tactique préétablie, enracinée dans l'entreprise et traduite en pratiques de gestion, y compris les pratiques de gestion des ressources humaines. Vountisjarvi (2006) mentionne également plusieurs groupes d'activités de RSE liées à la GRH, telles que les valeurs et l'éthique, la formation et le perfectionnement du personnel, la participation des employés, l'égalité des chances et la conciliation travail-vie personnelle. Par conséquent, il existe des liens étroits entre la RSE et la GRH car la RSE interne nécessite une dépendance à la GRH (Gond et al, 2011).

Selon Cornelius et al (2008), il est utile de différencier la RSE interne et externe, car de nombreuses entreprises ont tendance à négliger d'importants problèmes de ressources humaines internes tout en se concentrant sur le service à la communauté. La plupart des recherches sur la RSE impliquent les responsabilités de l'entreprise vis-à-vis des parties prenantes externes. Par conséquent, moins d'attention est accordée à la RSE interne (Cornelius et al, 2008). Cet article se concentre sur les activités de RSE internes, à l'exclusion de la RSE externe des entreprises. L'objectif principal de cette recherche est d'étudier l'influence du Leadership Participatif (LP) sur l'Identité Organisationnelle (IO) au moyen de la Responsabilité Sociale des Entreprises (RSE) perçue par les employés et de la Fierté d'Etre Membre (FEM) sous l'angle de la théorie du traitement de l'information sociale dans les PME béninoises.

L'association proposée du leadership participatif avec les perceptions internes des employés en matière de RSE est étayée par la théorie du traitement de l'information sociale (Salancik et Pfeffer, 1978). Cette théorie stipule que les personnes construisent leurs perceptions sur la base d'indices d'informations et de facteurs sociaux au-delà de l'impact de personnalités individuelles (Salancik et Pfeffer, 1978). Dans le contexte de l'emploi, les employés obtiennent des informations sur la nature de leur environnement de travail, ce qui les aide à rassembler des éléments et à développer leurs perceptions (Zafar, 2013). Pour Boekhorst (2015), les sources importantes d'informations sociales proviennent généralement des personnes jouissant d'un statut élevé ou d'un pouvoir élevé. Selon Zafar (2013), le leadership forme le contexte de travail des employés de manière significative, car il peut offrir des indices importants qui orientent la réflexion des employés. La théorie du traitement de l'information sociale explique que les perceptions des caractéristiques du travail ne sont pas fixes, mais plutôt affectées par les relations informationnelles dans lesquelles un individu est intégré (Salancik et Pfeffer, 1978). Boekhorst (2015) affirme que, en tant que source d'informations sociales de premier plan, les dirigeants ont une influence déterminante sur la perception par les employés des pratiques organisationnelles internes. En se référant à Lord et Maher (2002), le codage et la récupération d'informations sont deux étapes importantes du traitement de l'information et ont des implications importantes pour le leadership. Lorsqu'un individu code des informations, celles-ci sont simplifiées et transférées de la mémoire à court terme vers la mémoire à long terme. Lors de la phase de récupération, un individu rappelle les informations de la mémoire à long terme et émet un jugement. Lorsque des interactions se produisent entre le dirigeant et les employés, ceuxci encodent et interprètent certains signaux sociaux. Les interprétations orientent les perceptions des employés sur leur dirigeant et sur ses intentions et comportements qui, à leur tour, affecteront leurs perceptions à eux des pratiques organisationnelles (Zhang et al., 2011).

Bryman (2013) considère qu'un style de leadership participatif est censé générer des émotions positives pour les employés, car ils ont l'impression d'être entendus au sein de l'organisation. Dodge (1991) souligne que 
l'émotion est simplement pertinente pour traiter les informations d'une certaine manière. Par conséquent, en éprouvant des émotions positives avec un leadership participatif, les employés traiteraient l'information de manière positive et développeraient une perception positive des pratiques organisationnelles internes, y compris la RSE interne. De plus, lorsque les employés participent au processus de prise de décision, ils sont plus susceptibles de croire que leurs intérêts sont exprimés dans les actions de l'entreprise (Chen et Hung-Baesecke, 2014). La participation des employés leur permettra d'investir dans les actions qu'ils considèrent importantes et qui leur profiteraient finalement (Stawiski et al, 2010). Néanmoins, d'après Kolk et al (2010), le leadership participatif peut avoir des répercussions négatives sur les activités internes de la RSE, les leaders participatifs pouvant de ce fait discuter des attentes et des intérêts des employés dans leurs actions commerciales, amplifiant ainsi leur perception positive des pratiques de l'organisation. Par conséquent, sur la base de tout ce qui précède, le leadership participatif devrait conduire à une perception interne positive de la RSE des employés. Ainsi, l'hypothèse $\mathrm{H} 1$ est proposée :

H1 : Le leadership participatif aurait une relation positive avec les perceptions internes des employés en matière de RSE.

\section{E. Perceptions de la RSE et fierté d'être membre}

Une culture organisationnelle promouvant la RSE peut se manifester dans les émotions des employés, telles que la fierté (Onkila, 2015). Les employés sont plus susceptibles d'être fiers de leur appartenance à une entreprise lorsque celle-ci illustre des initiatives de RSE, principalement celles qui les concernent (Stawiski et al, 2010). Le concept de fierté a fait l'objet de beaucoup d'attention dans la recherche. Bein que la FEM soit reconnue pour son rôle important dans le contrôle des actions sociales quotidiennes, les recherches sur cet aspect restent insuffisantes (Yilmaz et al, 2015).

La relation proposée entre les perceptions internes de la RSE et la FEM est étayée par la théorie de l'identité sociale (TIS) (Tajfel et Turner, 1979) qui explique le processus par lequel les gens se perçoivent et se catégorisent en indiquant que les individus se classent dans différentes catégories sociales et voient leur appartenance à une catégorie spécifique sur la base de facteurs sociaux spécifiques (Tajfel et Turner, 1986). La théorie est connue pour former les racines de la fierté (Blader et Tyler, 2009). Selon la TIS, le groupe auquel appartiennent les individus est une source majeure de confiance et de fierté (Tajfel et Turner, 1979). Le caractère distinctif d'une entreprise donnée peut être démontré par ses initiatives de RSE qui l'identifient en partie (Sen et Bhattacharya, 2001) et l'importance de ces initiatives aux yeux des employés favorise la promotion de la FEM (Arnett et al, 2002). Lorsque l'entreprise révèle des valeurs significatives aux employés, ceux-ci sont plus susceptibles de se sentir fiers de leur appartenance (Reade, 2001). D'après De Roeck et al (2014) et Gond et al (2010), les employés développent des sentiments plus forts à partir des actions de RSE internes que des actions de RSE dirigées vers les divers groupes sociaux. Cependant, l'opinion des gens sur leur appartenance à une organisation n'est pas uniquement influencée par les perceptions personnelles de l'organisation, mais aussi par la manière dont les personnes extérieures à l'organisation la perçoivent (Brown et al, 2006 ; Peterson, 2004).

Les entreprises qui font preuve d'un comportement socialement responsable à l'égard de leurs employés reçoivent souvent des commentaires positifs de personnes extérieures à l'organisation pour le traitement réservé aux employés (Gond et al, 2010). Plusieurs études ont révélé que le facteur déterminant en matière d'évaluation de RSE d'une entreprise par le public repose sur sa perception du traitement équitable de ses employés (Googins et al, 2007 ; Rok, 2009). Les employés seront fiers d'appartenir à une organisation reconnue pour sa réputation de responsabilité sociale envers eux (Gond et al, 2010). Néanmoins, l'appartenance à des entreprises peu réputées en matière de traitement des employés peut engendrer des émotions négatives chez les employés, transformant leur fierté en un malaise et une gêne (Dutton et al, 1994). Ainsi, la fierté provient à la fois des autoévaluations et des opinions de personnes extérieures (Verbeke et al, 2004). 
En outre, le modèle de Lind et Tyler (1988) basé sur la valeur du groupe et celui de Tajfel et Turner, 1986) basé sur la TIS tiennent compte du fait que, lorsque des personnes perçoivent l'existence d'un traitement de haute qualité et de procédures équitables au sein de l'organisation, des sentiments positifs tels que la fierté et le respect, qui contribuent à leur estime de soi, sont développés (Tyler et al, 1996). Les perceptions de procédures organisationnelles impartiales montrent qu'une justice equitable au sein d'une organisation rend les employés plus valorisés par cette organisation et constituent des indicateurs majeurs de la capacité des personnes à être fières de leur appartenance à un certain groupe (Sousa et Vala, 2002). Cela indique que les perceptions positives des employés à l'égard de la RSE interne devraient générer des émotions positives telles que la fierté. Par conséquent, à partir de tout ce qui précède, l'hypothèse $\mathrm{H} 2$ est proposée :

H2 : Les perceptions internes de la RSE auraient une relation positive avec la fierté d'être membre.

Eu égard aux deux hypothèses émises plus haut, nous constatons que la RSE joue un rôle de médiateur dans la relation entre le leadership participatif et la FEM, ce qui permet de formuler l'hypothèse H3 :

H3 : La relation entre le leadership participatif et la FEM serait médiatisée par les perceptions internes de la RSE.

\section{F. Fierté d'être membre et identification organisationnelle}

Tracy et Robins (2007) affirment que la fierté est une émotion essentielle, car elle entraîne des conséquences importantes au quotidien. Elle a été suggérée dans la contribution de plusieurs concepts impératifs, en particulier pour l'IO (Sousa et Vala, 2002). Lorsque les individus se sentent fortement liés à leur entreprise, on pense que celle-ci est plus efficace et plus capable d'atteindre ses objectifs (Pratt, 1998 ; Van Dick et al., 2007). Par conséquent, il est important d'explorer le lien qui existe entre FEM et IO (Smidts et al., 2001).

L'IO est reconnue comme le processus psychologique fondamental qui sous-tend la théorie de l'identité sociale, car elle est considérée comme une forme explicite d'identification sociale (Ashforth et Mael, 1989). Selon la TIS, l'identification sociale est associée à plusieurs composantes affectives telles que la fierté, l'estime de soi et le concept de soi (Tajfel et Turner, 1986). Les sentiments découlant de l'appartenance à une organisation, tels que la fierté, sont depuis longtemps intégrés à la mise en œuvre opérationnelle d'IO. La TIS explique que l'IO des employés augmente lorsque la FEM est renforcée car, cela répond finalement au besoin d'appartenance des employés (De Roeck et Delobbe, 2012). En outre, lorsque les employés se sentent fiers de leur appartenance à une organisation, ils sont motivés pour s'identifier à l'entreprise afin de renforcer d'autres sentiments, tels que l'estime de soi (Ashforth et Mael, 1989). Le modèle de valeur du groupe (Lind et Tyler, 1988) suggère également que le respect et la fierté contribuent à la formation de l'identification afin de développer en conséquence des sentiments de confiance en soi. Cet article s'appuie sur le cadre théorique de Tyler et Blader $(2000 ; 2009)$ soutenant que, dans la mesure où le respect et la fierté sont liés au sentiment de confiance en soi et à l'estime de soi, ces valeurs sont essentielles à la formation d'identification avec leur entreprise.

Selon Hogg et Terry (2001), faire l'expérience de la FEM peut amener les employés à agir conformément aux valeurs et aux objectifs de l'entreprise. Par conséquent, lorsque les employés font l'expérience de la GIP, ils sont plus susceptibles d'internaliser les valeurs et les objectifs de l'entreprise (Van Knippenberg, 2000). Cette internalisation définit l'IO (Ekwutosi et Moses, 2013). Ainsi, la fierté aide directement les employés à s'identifier à leur organisation (Blader et Tyler, 2009). De plus, comme la fierté montre le jugement des gens sur le statut d'un groupe (Ines, 2012), la fierté que les employés obtiennent en travaillant dans une entreprise reconnue pour sa RSE interne renforce alors leur estime de soi et les encourage à s'identifier à l'organisation 
(Dutton et al., 1994). Par conséquent, les perceptions relatives aux pratiques de l'entreprise permettent aux employés de s'identifier indirectement à l'entreprise en renforçant leur FEM (Bergami et Bagozzi, 2000), ce qui permet de développer les deux hypothèses H4 et H5 :

H4 : La FEM serait positivement liée à l'identification organisationnelle.

H5 : La FEM médiatiserait la relation entre les perceptions internes des employés en matière de RSE et l'IO.

Sur la base des hypothèses ci-dessus, le modèle conceptuel suivant est développé :

\section{FIGURE II : LE MODÈLE CONCEPTUEL DE L’ÉTUDE}

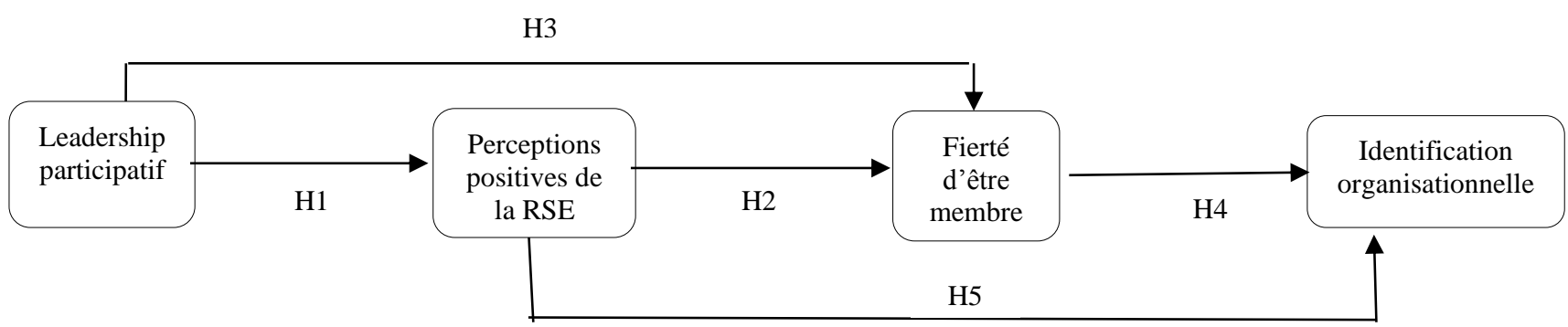

Variables indépendantes : Leadership participatif

Variables médiatrices : Perceptions positives de la RSE et FEM

Variables dépendantes : Identification Organisationnelle

\section{JUSTIFICATION DES CHOIX METHODOLOGIQUES}

Afin de confronter les éléments théoriques présentés jusqu'à présent à la réalité du terrain, nous avons mené une étude quantitative auprès de 740 employés de 17 PME opérant dans différents secteurs (le commerce de détail, la santé, la restauration, la téléphonie, l'agroalimentaire, les services, la distribution) et réparties dans trois villes au Bénin (5 à Cotonou, 7 à Porto-Novo et 5 à Parakou). Ces 17 PME constituent un échantillon théorique. Ces entreprises de taille et de branche d'activités volontairement différents, esquissent une série de variations qui nous ont permis de cerner les similarités et les différences de perceptions des personnes interrogées. Le caractère exploratoire de cette recherche, dû au faible nombre de travaux portant sur notre question de recherche, explique pour partie le choix méthodologique de l'étude de cas (Yin, 1989 ; Wacheux, 1996). Le recours à l'étude exploratoire se justifie dès lors que l'on doit définir un phénomène avec plus de précision, identifier les stratégies opportunes ou obtenir davantage d'éclaircissement avant de développer une approche (Charreire et Durieux, 2003). Les données recueillies auprès des différents individus travaillant dans ces différentes entreprises seront d'un grand apport pour notre recherche.

L'accès aux PME participantes a été facilité par le biais de contacts personnels. Les employés ont été priés de remplir un questionnaire accompagné d'une lettre de motivation expliquant les objectifs de la recherche, clarifiant le caractère volontaire de la participation et garantissant la confidentialité des réponses. A Cotonou, le questionnaire était transmis par les superviseurs à leurs subordonnés par courrier électronique interne. Les superviseurs des entreprises désignées à Porto-Novo et Parakou ont distribué les questionnaires à la main aux subordonnés. Dans chaque entreprise, une boîte de classement a été placée et dans laquelle les répondants avaient la possibilité de placer anonymement le questionnaire une fois rempli. Les boîtes ont ensuite été récupérées par une personne désignée à cet effet.

A Cotonou, sur les 475 questionnaires envoyés, 260 questionnaires utilisables ont été retournés, soit un taux de réponse de 54,74\%. Sur les 260 répondants, 56,54\% étaient des hommes. La majorité des participants avaient 
entre 31 et 40 ans $(32,31 \%)$ et entre 41 et 50 ans (31,92\%). En outre, 66,92\% des répondants ont déclaré que leur plus haut niveau de scolarité était un baccalauréat. La plupart des participants ont déclaré un mandat de 5 à 10 ans (36,54\%). A Porto-Novo, un total de 268 questionnaires utilisables sur les 420 questionnaires distribués a été retourné, ce qui a donné un taux de réponse de 63,81\%. Sur les 268 répondants, 60,07\% étaient des hommes. La majorité des participants avaient entre 18 et 30 ans $(27,24 \%)$ et entre 31 et 40 ans $(27,99 \%)$. En outre, $81,72 \%$ des répondants ont déclaré un baccalauréat comme leur plus haut niveau de scolarité. La plupart des participants ont déclaré un mandat de moins de 5 ans $(39,93 \%)$. Enfin, à Parakou, un total de 212 questionnaires utilisables sur les 415 questionnaires distribués a été retourné pour un taux de réponse de 51,08\%. Sur les 212 répondants, 58,02\% étaient des hommes. La majorité des participants avaient entre 31 et 40 ans $(32,08 \%)$. En outre, $77,83 \%$ des répondants ont déclaré que leur plus haut niveau de scolarité était un baccalauréat. La plupart des participants ont déclaré un mandat de moins de 5 ans $(41,04 \%)$.

Le questionnaire a été pré-testé par trois professionnels qui n'étaient pas impliqués dans l'étude. Les éléments du questionnaire sont mesurés sur une échelle de Likert avec des options de réponse allant de (1) « fortement en désaccord » à (7) «fortement en accord».

Une échelle à 6 items développée par Arnold et al (2000) a été utilisée pour mesurer le leadership participatif. Les exemples d'éléments comprennent «Mon superviseur écoute mes idées et mes suggestions » et «Mon superviseur donne à tous les membres du travail une chance d'exprimer leurs opinions ». Les participants ont été invités à se référer à leur superviseur direct. La fiabilité d'alpha de Cronbach de l'échelle des études menées à Cotonou, à Porto-Novo et à Parakou est de 0,91, 0,95 et 0,91, respectivement.

Une échelle à 8 éléments, choisie à partir de l'échelle de Maignan et Ferrell (2001), dérivée de la classification RSE de Carroll (1979) a été utilisée pour mesurer les perceptions de la RSE interne. La classification de Carroll (1979) est considérée comme utile pour examiner la manière dont la RSE est affichée dans un contexte de pays en développement (Visser, 2008). Maignan et Ferrell (2001) ont proposé plusieurs éléments pour mesurer les responsabilités économiques, juridiques, éthiques et discrétionnaires des entreprises proposées par Carroll (1979). Des conseils pour utiliser cette échelle ont été offerts après avoir contacté plusieurs responsables dans les trois villes considérées. Afin de rendre opérationnelle la définition interne de la RSE à partir de l'échelle existante, il était essentiel d'identifier les activités de l'entreprise qui caractérisent la RSE économique, juridique, éthique et discrétionnaire à l'égard des employés. Les éléments sélectionnés ont ensuite été comparés à une autre échelle de RSE populaire (Turker, 2009), qui comprend des éléments qui mesurent spécifiquement les perceptions des activités de RSE dirigées vers les employés. Les exemples de sujets incluent «Mon entreprise à des politiques internes qui empêchent la discrimination dans la rémunération et la promotion des employés» et «Mon entreprise soutient les employés qui souhaitent acquérir une formation supplémentaire ». La fiabilité d'alpha de Cronbach sur l'étendue des études à Cotonou, à Porto-Novo et à Parakou est de 0,91, 0,90 et 0,90, respectivement.

Une échelle à 6 items de Mael et Ashforth (1992) adaptée par Homburg et al (2009) a été utilisée pour mesurer l'IO. Les exemples de sujets incluent «Quand quelqu'un critique cette entreprise, cela ressemble à une insulte personnelle » et «Quand je parle de cette entreprise, je dis habituellement «nous » plutôt que « eux» ». La fiabilité alpha de Cronbach de l'échelle des études menées à Cotonou, à Porto-Novo et à Parakou vaut respectivement $0,88,0,92$ et 0,91 .

Une échelle de 4 items a été utilisée pour mesurer la FEM. Les sujets de cette échelle ont été développés par Cable et Turban (2003) et Helm (2013). Les exemples d'éléments comprennent « Je suis fier de m'identifier 
personnellement avec cette entreprise » et «Je suis fier lorsque d'autres m'associent à cette entreprise ». La fiabilité alpha de Cronbach de l'échelle des études à Cotonou, à Porto-Novo et à Parakou est respectivement de $0,84,0,91$ et 0,89 .

Les variables de contrôle comprennent le sexe, l'âge, le niveau d'instruction et la durée de l'emploi. Le genre était auparavant lié à la fierté au travail (Magee, 2015) et à l'IO (Aryee et Luk, 1996). On a également constaté que la fierté et l'IO évoluaient avec l'âge (Magee, 2015). De plus, il a été prouvé que la durée de la formation et la durée de l'organisation avaient un effet sur la fierté (Kraemer et Gouthier, 2014 ; O'Reilly et Chatman, 1986) et un lien significatif avec l'IO (Kreiner et Ashforth, 2004 ; Stinglhamber et al, 2015).

La modélisation par équation structurelle (MES) a été réalisée à l'aide du logiciel SPSS 21.0 afin de tester les hypothèses proposées. Le choix de cette méthode est basé sur la forte compétence de la MES pour traiter les variables latentes et leurs indicateurs observables, ainsi que pour comparer le modèle hypothétique aux données empiriques en fournissant les statistiques d'ajustement (Nachtigall et al, 2003). La MES est également connue pour simplifier l'analyse de médiation car il teste différentes hypothèses de médiation dans une seule analyse (MacKinnon, 2008). L'approche en deux temps suggérée par Anderson et Gerbing (1988) a été suivie. Le modèle de mesure qui spécifie la relation entre les éléments observés et leurs constructions correspondantes a d'abord été analysé afin de fournir une analyse de confirmation des validités convergentes et discriminantes (Campbell et Fiske, 1959). Par la suite, le modèle structurel qui spécifie les relations de cause à effet d'une construction à l'autre a été analysé afin d'évaluer la validité nomologique (Cronbach et Meehl, 1955).

\section{RESULTATS}

\section{A. Analyse de validité}

Le morcellement des sous-ensembles a été appliqué afin d'obtenir un meilleur rapport sujet-paramètre (Matsunaga, 2008). Les éléments avec les charges de facteur les plus élevées et les plus faibles ont été moyennés puis traités comme des indicateurs de leur construit respectif (Little et al., 2002). La RSE était composée de quatre parcelles, la direction participative et l'IO de trois parcelles, tans que la FEM se retrouvait avec deux parcelles.

Une analyse factorielle confirmatoire (CFA) a été appliquée pour vérifier dans quelle mesure les variables observées représentaient leur construit sous-jacent (Suhr, 2006). L'indice d'ajustement comparatif (CFI), l'indice de Tucker-Lewis (TLI), le résidu quadratique moyen standardisé (SRMR) et l'approximation de l'erreur quadratique moyenne (RMSEA) ont été utilisés pour tester la qualité de l'ajustement du modèle aux données. Une valeur CFI supérieure à 0,90, une valeur TLI supérieure à 0,80, une valeur SRMR inférieure à 0,08 et une valeur RMSEA inférieure à 0,10 ont été suggérées (Byrne, 2010). Les résultats de l'analyse CFA réalisée ont montré que le modèle de mesure correspond bien aux données des trois villes : $\chi^{2} / \mathrm{df}=3,50, \mathrm{CFI}=0,92$, TLI = $0,88, \mathrm{SRMR}=0,03$, et RMSEA $=0,09$ pour Cotonou $; \chi^{2} / \mathrm{df}=3,11, \mathrm{CFI}=0,95, \mathrm{TLI}=0,93, \mathrm{SRMR}=0,03$, et RMSEA $=0,08$ pour Porto-Novo ; puis $\chi^{2} / \mathrm{df}=2,82, \mathrm{CFI}=0,93, \mathrm{TLI}=0,90, \mathrm{SRMR}=0,05$, et $\mathrm{RMSEA}=0,09$ pour Parakou. En outre, les résultats de la CFA ont révélé que les scores de fiabilité composites des variables allaient de 0,80 à 0,96, dépassant ainsi la valeur seuil recommandée de 0,70 (Nunnally, 1994). De même, conformément aux directives de Fornell et Larcker (1981), la variance moyenne extraite (AVE) pour chaque variable dépassait 0,5 , variant entre 0,66 et 0,89 . Ainsi, la validité convergente est prise en charge. Le tableau I présente les matrices de corrélation pour les trois échantillons. 
NAKOU et al.: Leadership participatif et identification organisationnelle : Rôle médiateur de la ...

TABLEAU I : CORRÉLATION ENTRE LES ÉTUDES

\begin{tabular}{|c|c|c|c|c|c|c|c|c|c|}
\hline & & 1 & 2 & 3 & 4 & 5 & 6 & 7 & 8 \\
\hline \multicolumn{10}{|c|}{ Variables dans l'échantillon de Cotonou } \\
\hline 1 & Genre & & & & & & & & \\
\hline 2 & Age &,$- 29 * *$ & & & & & & & \\
\hline 3 & Education & ,08 &,$- 19 * *$ & & & & & & \\
\hline 4 & Mandat &,$- 19 * *$ &, $71 * *$ &,$- 24 * *$ & & & & & \\
\hline 5 & LP &, 06 &, 06 & ,09 & 07 & $, \mathbf{8 8},(, 91)$ & & & \\
\hline 6 & RSE &, 11 &, 01 &, $20 * *$ &,- 12 &, $52 * *$ & $\mathbf{8 5},(, 91)$ & & \\
\hline 7 & FEM &, $19 * *$ &, 08 &, $19 * *$ &,- 05 &, $55^{* *}$ &, $68 * *$ & $, \mathbf{8 2},(, 80)$ & \\
\hline 8 & IO & ,09 &, 09 &, $16^{*}$ &, 11 &, $66 * *$ &, $73 * *$ &, $81 * *$ & $, \mathbf{8 4},(, 88)$ \\
\hline \multicolumn{10}{|c|}{ Variables dans l'échantillon de Porto-Novo } \\
\hline 1 & Genre & & & & & & & & \\
\hline 2 & Age &,- 09 & & & & & & & \\
\hline 3 & Education &,- 01 &,$- 24 * *$ & & & & & & \\
\hline 4 & Mandat &,- 06 &, $82 * *$ &,$- 31 * *$ & & & & & \\
\hline 5 & LP &,- 05 &, 03 &, 12 &,- 10 & ,94, (,96) & & & \\
\hline 6 & RSE &, 08 &,- 1 & ,07 &,$- 22 * *$ &, $50 * *$ & ,83,(,90) & & \\
\hline 7 & FEM &,- 003 &,- 09 &, $12 *$ &,$- 26 * *$ &, $63 * *$ &, $55^{* *}$ & ,95,(,95) & \\
\hline 8 & IO &, 01 &,- 41 &, 11 &, $12 *$ &, $78^{* *}$ &, $62 * *$ &, $72 * *$ & ,90,(,93) \\
\hline \multicolumn{10}{|c|}{ Variables dans l'échantillon de Parakou } \\
\hline 1 & Genre & & & & & & & & \\
\hline 2 & Age &,- 09 & & & & & & & \\
\hline 3 & Education &, 02 &,$- 18 * *$ & & & & & & \\
\hline 4 & Mandat &,- 03 &, $79 * *$ &,$- 27 * *$ & & & & & \\
\hline 5 & LP &,- 09 &, 01 &, 07 &,- 11 & $, 88,(, 91)$ & & & \\
\hline 6 & RSE &, 08 &,$- 23 * *$ & ,06 &,$- 21 * *$ &, $35 * *$ & ,83,(,90) & & \\
\hline 7 & FEM &,- 03 &, 01 &, 08 &,- 12 &, $62 * *$ &, $38 * *$ &, $\mathbf{8 7 , ( , 8 6 )}$ & \\
\hline 8 & IO &,- 05 &,- 10 & ,08 &,- 07 &, $74 * *$ &, $58 * *$ &, $58 * *$ & $, 88,(, 91)$ \\
\hline
\end{tabular}

Les chiffres en gras dans les diagonales des matrices représentent les racines carrées des AVE des constructions. La validité discriminante est atteinte si la racine carrée de l'AVE d'une construction est supérieure aux corrélations entre cette construction et d'autres constructions (Fornell et Larcker, 1981). Dans les trois matrices, ces racines carrées sont plus grandes que les corrélations entre la construction donnée et les autres constructions. Cela confirme la validité discriminante des échelles utilisées.

\section{B. Biais de méthode commune}

Afin de résoudre le problème de biais de la méthode commune, les remèdes recommandés par Podsakoff et al (2003) ont été suivis. Pendant la collecte des données, les réponses des répondants étaient gênées par le secret et la confidentialité des réponses afin de réduire l'appréhension de l'évaluation et la désirabilité sociale de l'évaluation. De plus, l'impact potentiel du biais de la méthode commune a été testé statistiquement à l'aide du test à facteur unique de Harman. Les statistiques 
d'ajustement du modèle à facteur unique étaient les suivantes $: \chi^{2} / \mathrm{df}=4,47, \mathrm{GFI}=0,72, \mathrm{CFI}=0,78$, $\mathrm{SRMR}=0,33$, et RMSEA $=0,12$ pour Cotonou $; \chi 2 / \mathrm{df}=3,84, \mathrm{GFI}=0,75, \mathrm{CFI}=0,85, \mathrm{SRMR}=0,40$, et $\mathrm{RMSEA}=0,10$ pour Porto-Novo ; et $\chi^{2} / \mathrm{df}=3,00, \mathrm{GFI}=0,75, \mathrm{CFI}=0,83, \mathrm{SRMR}=0,13$, et RMSEA $=0,10$ pour Parakou. Celles-ci indiquent un modèle inadéquat pour les trois villes, ce qui suggère que le biais de méthode commune n'est pas un problème important dans cette recherche. Pour plus de confirmation, étant donné que la tendance des personnes à se montrer positives ou « socialement souhaitables » lorsqu'on remplit des questionnaires est souvent considérée comme une cause de la variance commune des méthodes, la procédure de corrélation partielle a été appliquée en favorisant la désirabilité sociale. Cette procédure statistique nécessite que le chercheur contrôle les effets de la variance de la méthode commune (en contrôlant la désirabilité sociale), puis compare les différences de corrélation partielle entre les différentes variables (Posdakoff et al, 2003). Les réponses socialement souhaitables ont été mesurées à l'aide d'une échelle à 4 items développée par Donavan et al (2004). Les inspections des corrélations partielles après contrôle de l'opportunité sociale ont suggéré qu'il y avait un léger changement dans la force de certaines des corrélations. Pour la ville de Cotonou, les corrélations n'ont que très peu changé entre les variables. Par exemple, la corrélation entre les perceptions internes de la RSE et l'IO s'est accrue de 0,01. De même, pour les villes de Porto-Novo et Parakou, les corrélations entre les différentes variables ont légèrement changé. Cela signifie que la prise en compte de la réponse à la désirabilité sociale n'a eu que très peu d'effet sur la force des relations entre les variables, traduisant ainsi la faiblesse de la probabilité d'un biais de méthode commune dans les données de l'étude.

\section{Tests d'hypothèses}

Une estimation par maximum de vraisemblance a été réalisée pour tester les hypothèses proposées. La figure III, qui représente le modèle de médiation séquentielle partielle, montre les résultats de l'analyse SEM pour les trois villes d'étude, respectivement. Comme on le voit, le chemin qui mène du leadership participatif aux perceptions de la $\operatorname{RSE}(5=0,52, \mathrm{p}<0,01$ pour la ville de Cotonou ; $5=0,50$, $\mathrm{p}<0,01$ pour la ville de Porto-Novo $5=0,35, \mathrm{p}<0,01$ pour la ville de Parakou) est positif et significatif. Cela fournit des preuves à l'appui de l'hypothèse H1, selon laquelle le leadership participatif a une relation positive avec les perceptions internes des employés en matière de RSE. De même, le chemin des perceptions internes de la RSE vers la FEM $(5=0,51$ pour Cotonou/0,28 pour Porto-Novo/0,21 pour Parakou ; $\mathrm{p}<0,01)$ et le chemin entre la FEM et l'IO $(5=0,51, \mathrm{p}<0,01$ pour la ville de Cotonou 5 $=0,31, \mathrm{p}<0,01$ pour la ville de Porto-Novo, $5=0,14, \mathrm{p}<0,05$ pour la ville de Parakou) est positif et significatif. Cette démarche soutient l'hypothèse H2 postulant que les perceptions de la RSE sont positivement liées à la FEM et l'hypothèse H4 suggère que la FEM est positivement liée à la l'IO. Prises ensemble, les conclusions de la figure III illustrent la présence d'une certaine médiation, corroborant les hypothèses $\mathrm{H} 3$ et $\mathrm{H} 5$, selon lesquelles la relation entre le leadership participatif et la gestion des informations personnelles est influencée par les perceptions internes des employés en matière de RSE et par le lien qui existe entre elles, est médiée par la FEM. 


\section{FIGURE III : RÉSULTATS DU MODÈLE D’ÉQUATIONS STRUCTURELLES}

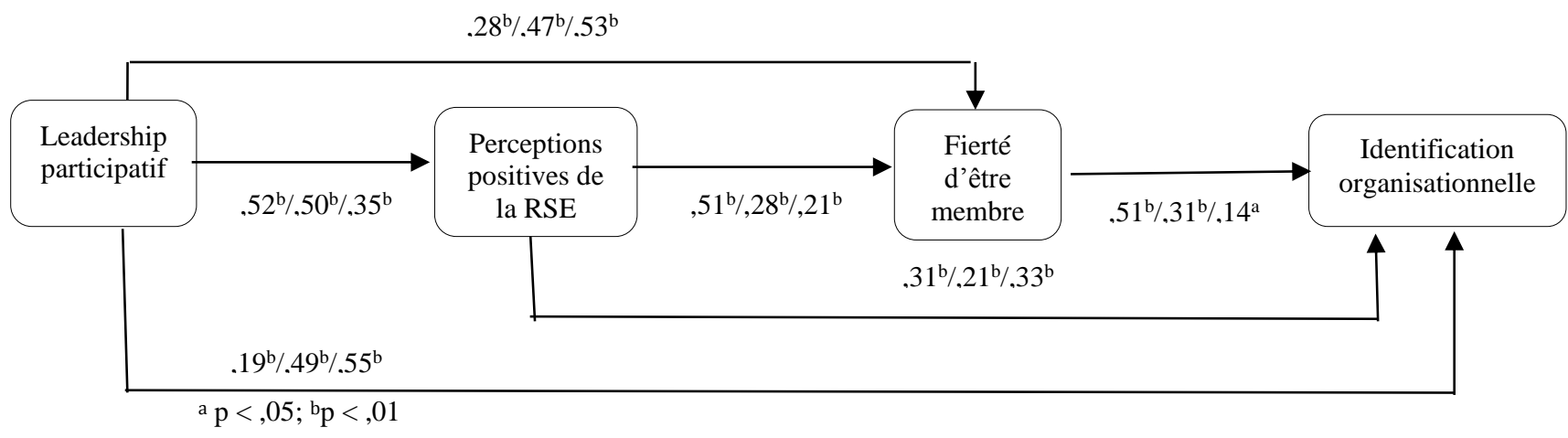

\section{Analyse de médiation}

Une comparaison entre un modèle alternatif, qui permet uniquement une médiation complète, et le modèle de médiation partielle, a été effectuée. Le modèle entièrement médiatisé (Figure III) a montré l'ajustement suivant : Pour la ville de Cotonou, $\chi^{2}(91)=361,68, \chi^{2} / \mathrm{df}=3,97, \mathrm{CFI}=0,89$, TLI $=0,86, \mathrm{SRMR}=0,09$, et RMSEA $=0,11$; pour la ville de Porto-Novo, $\chi^{2}(91)=453,08, \chi^{2} / \mathrm{df}=4,98$, $\mathrm{CFI}=0,89$, TLI $=0,86, \mathrm{SRMR}=0,14$, et RMSEA $=0,12$; et pour Parakou, $\chi^{2}(91)=409,98, \chi^{2} / \mathrm{df}=$ $4,51, \mathrm{CFI}=0,84, \mathrm{TLI}=0,80, \mathrm{SRMR}=0,17$, et $\mathrm{RMSEA}=0,13$.

Le modèle de médiation partielle a montré un meilleur ajustement aux données des trois villes d'étude, respectivement $: \chi^{2}(88)=306,44 / 278,76 / 247,75 ; \chi^{2} / \mathrm{df}=3,48 / 3,16 / 2,81 ; \mathrm{CFI}=$ 0,91/0,94/0,92; TLI = 0,89/0,93/0,90 ; SRMR = 0,06/0,06/0,07 ; et RMSEA = 0,09/0,09/0,09. La différence d'ajustement était statistiquement significative : $\Delta \chi^{2}(3)=55,24 / 174,32 / 162,23, \mathrm{p}<0,01$ pour les trois villes d'étude.

De surcroît, l'amorçage a été utilisé pour valider les résultats de médiation du SEM. Suivant les directives recommandées par Shrout et Bolger (2002), 5000 échantillons bootstrap ont été créés pour chaque ville. Les effets indirects du leadership participatif sur la FEM et les effets indirects des perceptions internes de la RSE sur l'IO ont été estimés avec la méthode des percentiles à correction de biais (Efron et Tibshirami, 1993). Les résultats de l'échantillon de démarrage à partir du fichier de la ville de Cotonou révèlent que l'effet indirect du leadership participatif sur la FEM via les perceptions internes de la RSE est de 0,27, obtenu en multipliant les coefficients de chemin du LP vers la RSE $(\beta=0,52)$ et de RSE vers FEM $(\beta=0,51)$. Cet effet indirect est significatif avec un $\mathrm{p}<0,01$ et un intervalle de confiance (IC) correspondant à $95 \%$ compris entre 0,10 et 0,36 . En outre, l'effet indirect des perceptions internes de la RSE sur l'IO via la FEM pour Cotonou est 0,26, obtenu en multipliant les coefficients de chemin de RSE par ceux de la FEM $(\beta=0,51)$ et de la FEM par ceux de l'IO $(\beta=0,51)$. Cet effet indirect est significatif avec un $\mathrm{p}<0,01$ et un IC à $95 \%$ compris entre 0,08 et 0,48 . De même, les résultats de l'échantillon bootstrap de Porto-Novo montrent que l'effet indirect du leadership participatif sur la FEM via les perceptions internes de la RSE est de 0,14 $\left(\beta_{\mathrm{LP} \rightarrow \mathrm{RSE}}=0,50^{*} ; \beta_{\mathrm{RSE} \rightarrow \mathrm{FEM}}=0,28\right)$. Cet effet indirect est significatif $(\mathrm{p}<0,01)$ avec un IC à $95 \%$ 
compris entre 0,04 et 0,24. L'effet indirect des perceptions internes de la RSE sur l'IO via la FEM pour la ville de Porto-Novo est de $0,09\left(\beta_{\mathrm{RSE} \rightarrow \mathrm{FEM}}=0,28^{*} \beta_{\mathrm{FEM} \rightarrow \mathrm{IO}}=0,31\right)$. Cet effet est significatif $(\mathrm{p}<0,01)$ avec un IC à $95 \%$ des valeurs allant de 0,03 à 0,20 . En appliquant les mêmes processus, les résultats de l'échantillon bootstrap de la ville de Parakou montrent que l'effet indirect du leadership participatif sur la FEM via les perceptions internes de la RSE est de $0,07\left(\beta_{\mathrm{LP} \rightarrow \mathrm{RSE}}=0,35^{*} \beta_{\mathrm{RSE} \rightarrow \mathrm{FEM}}\right.$ $=0,21)$. Il est significatif avec un $\mathrm{p}<0,01$ et un IC à $95 \%$ compris entre 0,01 et 0,25 . Enfin, l'effet indirect des perceptions internes de la RSE sur l'IO via la FEM pour la ville de Parakou est de 0,03 $\left(\beta_{\mathrm{RSE} \rightarrow \mathrm{FEM}}=0,21^{*} \beta_{\mathrm{FEM} \rightarrow \mathrm{IO}}=0,14\right)$, et est significatif avec un $\mathrm{p}<0,1$. Par conséquent, les résultats présentés soutiennent ceux des hypothèses de médiation.

\section{DISCUSSION}

Au plan théorique, cet article atteste l'influence du LP sur l'IO au moyen de la RSE perçue par les employés et de la FEM dans les PME béninoises. Il part de la théorie de l'information sociale au sens de Tajfel et Turner (1986) pour expliquer l'influence réciproque de la relation entre le LP sur l'IO. Il élargit également le champ de compréhension du modèle attitude-intention-comportement de Bagozzi (1992) et de la théorie des attachements affectifs de Lawler (1992) dans le contexte de la RSE des PME et des employés.

$\mathrm{Au}$ plan méthodologique, cet article utilise une démarche méthodologique quantitative pour répondre aux critiques formulées contre les modèles qui tentent d'expliquer et de conceptualiser le LP, la RSE, la FEM et l'IO.

Au plan managérial, cet article montre que le LP affecte l'IO des employés des PME au Bénin. Il met en lumière implicitement les stratégies et les initiatives à développer par les managers pour susciter des émotions et des comportements positifs au travail pouvant accroître l'identification organisationnelle des employés.

Malgré l'importance croissante de l'influence que le leadership participatif peut avoir sur les employés, ce style de leadership n'a pas été lié à l'IO. En outre, les mécanismes par lesquels un leadership participatif peut aboutir à des résultats souhaités par les employés manquent d'examen (Hassan et al, 2013 ; Miao et a, 2014). Le but de cette étude était de déterminer si et comment un leadership participatif conduit à une IO plus élevée. Les résultats ont montré que le leadership participatif finit par générer des identifications organisationnelles. Les résultats ont confirmé l'hypothèse voulant que le leadership participatif conduise à une perception interne positive de la RSE chez les employés. Les résultats ont également corroboré les hypothèses suggérant que les perceptions positives de la RSE interne conduisent à la FEM, qui à son tour mène à l'IO. En outre, des éléments de preuve ont été fournis pour les hypothèses indiquant que les perceptions internes de la RSE médiatisent la relation entre le LP et la FEM, et que cette dernière médie la relation entre les perceptions internes de la RSE et l'IO. Néanmoins, les relations de médiation se sont révélées partielles, laissant entrevoir l'existence d'autres médiateurs dignes d'enquête. Par exemple, un niveau élevé de confiance dans le leader peut jouer un rôle de médiateur dans la relation entre le leadership participatif et la FEM, car un leadership participatif peut démontrer que le leader a du respect, de la préoccupation et de la confiance envers les employés (Dirks et Ferrin, 2002 ; Miao et al, 2013). 
D'autres mécanismes peuvent également être impliqués dans la relation entre la RSE et l'IO, tels que le degré d'importance du travail d'un individu pour sa propre image, car on pense que les employés s'identifient davantage à l'organisation lorsqu'ils considèrent que leur travail redore leur image de soi (Katrinli et al, 2009).

Les relations étudiées sont les plus fortes pour la ville de Cotonou, suivies de celles de Porto-Novo puis de celles de Parakou. Les dirigeants de la ville de Cotonou sont censés déployer des efforts supplémentaires pour communiquer et écouter les besoins de leurs subordonnés, car la gestion de la diversité actuelle en milieu de travail est devenue une priorité pour de nombreuses entreprises (AlJenaibi, 2012). Un type particulier de relation basé sur le respect mutuel et la confiance est en train de naître entre les divers employés et les dirigeants des entreprises de Cotonou afin de générer avec succès des résultats de travail positifs (Suliman et Al-Kathairi, 2012). De plus, les différents partis religieux et politiques de la ville de Porto-Novo ont adopté une posture de tolérance et de compréhension des éléments particuliers de la coexistence positive sur le lieu de travail (Jamali et al, 2005).

La découverte que le leadership participatif conduit à des perceptions internes positives des employés en matière de RSE apporte un soutien à la théorie du traitement de l'information sociale. Cette constatation est cohérente avec l'idée que les employés développent leurs perceptions des pratiques organisationnelles internes en collectant des informations provenant de leur environnement de travail et que leur dirigeant constitue une source importante d'informations (Salancik et Pfeffer, 1978). Aussi, la découverte contribute-t-elle à la théorie du traitement de l'information sociale en élargissant la compréhension de cette théorie dans un contexte d'emploi. Cette découverte contribue également à élargir le noyau de la théorie de la RSE en déterminant que le leadership participatif est un antécédent des perceptions internes de la RSE, et en examinant la RSE dans le contexte des PME des pays en développement, car elle est pertinente mais insuffisante (Jamali et al, 2015 ; Spence, 2014). Ces constatations vont dans le sens de l'idée selon laquelle l'identification est façonnée par la perception qu'ont les employés de l'organisation et de ses pratiques, ce qui suscite un sentiment de fierté chez les employés (Tajfel et Turner, 1979).

\section{CONCLUSION}

Dans le but d'etudier le processus reliant le leadership participatif (LP) à l'identification organisationnelle (IO) et de déterminer un nouvel antécédent de perception de la responsabilité sociale des entreprises (RSE), un modèle de médiation séquentielle a été élaboré et testé dans les PME béninoises. Les résultats ont démontré que le leadership participatif conduit à des perceptions internes positives des employés en matière de RSE, convergeant vers la fierté d'être member (FEM) qui, à son tour, aboutit à une IO. En outre, les perceptions internes de la RSE ont servi de médiation à la relation entre le leadership participatif et la FEM, et la FEM, de médiation entre les perceptions internes de la RSE et l'IO à Cotonou, à Porto-Novo et à Parakou. 
Les résultats de cette recherche offrent un regard sur les dirigeants d'entreprise en quête d'une IO plus élevée de la part de leurs employés. Il est bien connu que les employés qui s'identifient fortement à leur entreprise affichent des attitudes et des comportements positifs sur le lieu de travail, qui aident l'entreprise à atteindre ses objectifs et à être plus efficace (Pratt, 1998 ; Van Dick et al, 2007). Par conséquent, les gestionnaires peuvent adopter une approche plus participative en interagissant avec leurs subordonnés afin de favoriser un état psychologique positif des employés où ils se sentent fiers de leur appartenance à l'organisation et s'identifient ainsi à elle. De même, les perceptions positives de la RSE interne dans la suite entraînant une augmentation de l'IO, une modification des pratiques de gestion des ressources humaines peut profiter à la fois à l'entreprise et aux employés (Fajana et al, 2011). Par exemple, intégrer des politiques internes qui empêchent la discrimination dans la promotion et la rémunération des employés si ces pratiques ne sont pas encore présentes ou qui aident les employés souhaitant acquérir une formation supplémentaire. Cette option aura de la valeur pour les employés. De telles pratiques, considérées comme des éléments de mesure de la RSE interne, renforceront également la perception positive des employés à l'égard de la RSE interne de l'entreprise, augmentant ainsi leur IO et bénéficiant ainsi à l'entreprise (Gond et al, 2010 ; Turker, 2009).

Malgré des considérations soigneuses, l'article présente certaines limites. Premièrement, en raison du contexte de l'étude, l'aspect externe de la RSE n'a pas été pris en compte car les trois villes d'étude manquent toujours d'initiatives répondant aux besoins des différentes parties prenantes (Shehadi et al, 2013). Des recherches futures pourront tester le modèle de médiation séquentielle de cet article dans d'autres villes béninoises où les perceptions externes de la RSE sont également prises en compte. En outre, les résultats de la recherche ont révélé des relations de médiation partielles et, par conséquent, une extension de la recherche consiste à tester d'autres mécanismes dans la relation entre LP et FEM ainsi que dans la relation entre RSE et IO, tels que des niveaux élevés de confiance dans le leader et le degré d'importance du travail pour l'image de soi de l'individu. Et puisque cette recherchee n'est que transversale, les recherches futures pourront être complétées par une étude longitudinale permettant de suivre et de décrire les changements individuels au fil du temps (Menard, 2002). En dépit de ces limites, cette étude a fourni des résultats intrigants qui traitent des lacunes de la littérature et améliorent les connaissances en ce qui concerne le processus conduisant à une IO plus élevée.

\section{REFERENCES}

[1] Aguilera R.V., Rupp D.E., Williams C.A. \& Ganapathi, J. Putting the S back in corporate social responsibility: A multilevel theory of social change in organizations. Academy of Management Review, 2007, 32(3), 836-863.

[2] Anderson J.C. \& Gerbing D.W. Structural equation modeling in practice : a review and recommended two-step approach. Psychological Bulletin, 1988, 103(3), 411-423.

[3] Ashforth B.E. \& Mael F. Social identity theory and the organization. Academy of Management Review, 1989, 14(1), $20-39$.

[4] Ayse K. Y., Imran A. \& Triant F. The effect of corporate social responsibility on pride in membership, job satisfaction and employee engagement. British Journal of Economics, Management \& Trade, 2015, 9(4), 1-12.

[5] Basil D.Z. \& Erlandson J. Corporate social responsibility website representations: A longitudinal study of internal and external self - presentations. Journal of Marketing Communications, 2008, 14(2), 125-137.

[6] Bergmann A., Schmidt S.L., Schreyer, D. \& Torgler B. Age and organizational identification : empirical findings from professional sports. Applied Economics Letters, 2016, 23(10), 718-722. 
[7] Bhattacharya C.B., Korschun D. \& Sen, S. Strengthening stakeholder-company relationships through mutually beneficial corporate social responsibility initiatives. Journal of Business Ethics, 2009, 85(2), 257-272.

[8] Boekhorst J.A. The role of authentic leadership in fostering workplace inclusion : A social information processing perspective. Human Resource Management, 2015, 54(2), 241-264.

[9] Boekhorst J.A. The role of authentic leadership in fostering workplace inclusion : A social information processing perspective. Human Resource Management, 2015, 54(2), 241-264.

[10] Breuer A., Landman, T. \& Farquhar D. Social media and protest mobilization: Evidence from the Tunisian revolution. Democratization, 2015, 22(4), 764-792.

[11] Bryman A. Leadership and organizations. Routledge, 2013.

[12] Byrne B. M. Structural equation modeling with AMOS : Basic concepts, applications, and programming (2nd ed.). New York : Routledge Academy, 2010.

[13] Cable D. \& Turban D.B. The value of organizational reputation in the recruitment context: a brand-equity perspective. Journal of Applied Social Psychology, 2003, 33(11), 2244-2266.

[14] Carmeli A., Gilat G. \& Waldman D.A. The role of perceived organizational performance in organizational identification, adjustment and job performance. Journal of Management Studies, 2007, 44(6), 972-992.

[15] Carroll A.B. A three-dimensional conceptual model of corporate performance. Academy of Management Review, 1979, 4(4), 497-505.

[16] Chen Y.R. \& Hung-Baesecke C.F. Examining the internal aspect of corporate social, 2014.

[17] Choi Y. \& Yu Y. The influence of perceived corporate sustainability practices on employees and organizational performance. Sustainability, 2014, 6(1), 348-364.

[18] Choudhary A.I., Akhtar, S.A. \& Zaheer, A. Impact of transformational and servant leadership on organizational performance : A comparative analysis. Journal of Business Ethics, 2013, 116(2), 433-440.

[19] De Roeck K., Marique G., Stinglhamber F. \& Swaen V. Understanding employees' responses to corporate social responsibility: mediating roles of overall justice and organisational identification. The International Journal of Human Resource Management, 2014, 25(1), 91-112.

[20] Ekwutosi O. C. \& Moses O. S. Internalization of organizational culture : a theoretical perspective. International Journal of Business Tourism and Applied Sciences, 2013, 1(2), 77-96.

[21] Gond J.P., El-Akremi A., Igalens J. \& Swaen V. Corporate social responsibility influence on employees. Research Paper Series. International Centre for Corporate Social Responsibility. Nottingham University, 2010.

[22] Gond J.P., Igalens J., Swaen V. \& El Akremi A. The human resources contribution to responsible leadership : An exploration of the CSR-HR interface. Journal of Business Ethics, 2011, 98(1), 115-132.

[23] Habib R. R. Syrian labor in Lebanon : refugees and workers. In 2015 APHA Annual Meeting \& Expo (Oct.-Nov. 2015). APHA.

[24] Hall, B.H., Lotti, F., \& Mairesse, J. Innovation and productivity in SMEs: empirical evidence for Italy. Small Business Economics, 2009, 33(1), 13-33.

[25] Hassan S., Mahsud R., Yukl G. \& Prussia G. E. Ethical and empowering leadership and leader effectiveness. Journal of Managerial Psychology, 2013, 28(2), 133-146.

[26] Helm S. A matter of reputation and pride: Associations between perceived external reputation, pride in membership, job satisfaction and turnover intentions. British Journal of Management, 2013, 24(4), 542-556.

[27] Huang X., Iun J., Liu A. \& Gong Y. Does participative leadership enhance work performance by inducing empowerment or trust ? The differential effects on managerial and non - managerial subordinates. Journal of Organizational Behavior, 2010, 31(1), $122-$ 143.

[28] Jamali D. R., El Dirani A. M. \& Harwood I. A. Exploring human resource management roles in corporate social responsibility : the CSR - HRM co - creation model. Business Ethics: A European Review, 2015, 24(2), 125-143.

[29] Jong P.J., \& Hartog D. D. How leaders influence employees' innovative behavior. European Journal of Innovation Management, 2007, 10(1), 41-64. 
[30] Kraemer T. \& Gouthier M. How organizational pride and emotional exhaustion explain turnover intentions in call centers : A multi-group analysis with gender and organizational tenure. Journal of Service Management, 2014, 25(1), 125-148.

[31] Kreiner G.E. \& Ashforth B.E. Evidence toward an expanded model of organizational identification. Journal of Organizational Behavior, 2004 25(1), 1-27.

[32] Lee E. M., Park S. Y. \& Lee H. J. Employee perception of CSR activities : Its antecedents and consequences. Journal of Business Research, 2013, 66(10), 1716-1724.

[33] Lind E. A. \& Tyler, T. R. The social psychology of procedural justice. New York : Plenum, 1988.

[34] MacCallum R.C., Browne M.W. \& Sugawara H.M. Power analysis and determination of sample size for covariance structure modeling. Psychological Methods, 1996, 1(2), 130-149.

[35] MacKinnon D. Introduction to statistical mediation analysis. New York, NY : Lawrence Erlbaum Associates, 2008.

[36] Mael F. \& Ashforth, B.E. Alumni and their alma mater : A partial test of the reformulated model of organizational identification. Journal of Organizational Behavior, 1992, 13(2), 103-123.

[37] Magee W. Effects of Gender and Age on Pride in Work, and Job Satisfaction. Journal of Happiness Studies, 2015, 16(5), 10911115 .

[38] Martin R. A review of the literature of the followership since 2008. SAGE Open, 2015, 5(4), 1-9.

[39] McWilliams A. \& Siegel D. S. Corporate social responsibility: A theory of the firm perspective. Academy of Management Review, 26(1), 2001, 117-126.

[40] Miao Q., Newman A. \& Huang X.The impact of participative leadership on job performance and organizational citizenship behavior: Distinguishing between the mediating effects of affective and cognitive trust. The International Journal of Human Resource Management, 2014, 25(20), 2796-2810.

[41] Miao Q., Newman A., Schwarz G. \& Xu L. Participative leadership and the organizational commitment of civil servants in China : the mediating effects of trust in supervisor. British Journal of Management, 2013, 24(1), 76-92.

[42] Onkila T. Pride or embarrassment ? Employees' emotions and corporate social responsibility. Corporate Social Responsibility and Environmental Management, 2015, 22(4), 222-236.

[43] O'Reilly C. \& Chatman J. Organizational commitment and psychological attachment: The effects of compliance, identification and internalization on prosocial behavior. Journal of Applied Psychology, 1986, 71(3), 492-499.

[44] Salancik G. R. \& Pfeffer J. A social information processing approach to job attitudes and task design. Administrative Science Quarterly, 1978, 23(2), 224-253.

[45] Salancik, G. R. \& Pfeffer, J. A social information processing approach to job attitudes and task design. Administrative Science Quarterly, 1978, 23(2), 224-253.

[46] Shin I., Hur, W. M. \& Kang, S. Employees' perceptions of corporate social responsibility and job performance : A sequential mediation model. Sustainability, 2016, 8(5), 493.

[47] Somech, A. Directive versus participative leadership : Two complementary approaches to managing school effectiveness. Educational Administration Quarterly, 2005, 41(5), 777-800.

[48] Stanton S. Equality and Justice in Employment : A Case Study from Post-Revolution Tunisia. The Equal Rights Review, 2015, $15(1), 112-127$.

[49] Stawiski S., Deal, J. J. \& Gentry, W. Employee perceptions of corporate social responsibility. Center for Creative Leadership, Greensboro, NC, 2010..

[50] Suliman A. M. \& Sumina T. Leadership and CSR in developing countries : the case of the UAE. Corporate Social Responsibility and Human Resource Management : A Diversity Perspective, 2014, 250-266.

[51] Tajfel H. \& Turner J. C. An integrative theory of intergroup conflict. The Social Psychology of Intergroup Relations, 1979, $33(47), 33-47$.

[52] Tajfel H. \& Turner, J. C. The social identity theory of intergroup behavior. In S. Worchel \& W. G. Austin (Eds.), Psychology of intergroup relations (2nd edn., 1986, 7-24), Chicago : Nelson Hall.

[53] Turker D. Measuring corporate social responsibility: A scale development study. Journal of Business Ethics, 2009, 85(4), 411427. 
[54] Tyler T., \& Blader S.L. Cooperation in groups : Procedural justice, social identity, and behavioral engagement. Philadelphia : Psychology Press, 2000.

[55] Vázquez-Carrasco R. \& López-Pérez M. E. Small \& medium-sized enterprises and corporate social responsibility : a systematic review of the literature. Quality \& Quantity, 2013, 47(6), 3205-3218.

[56] Vlachos P. A., Panagopoulos N. G. \& Rapp, A. A. Employee judgments of and behaviors toward corporate social responsibility: A multi - study investigation of direct, cascading, and moderating effects. Journal of Organizational Behavior, 2014, 35(7), 9901017.

[57] Zafar A. Linking Ethical Leadership and Employees' In-Role Performance : Exploring the Mediating roles of Psychological Capital and Follower-Leader Relational Capital. Brock University, 2013.

[58] Zhu Y., Sun L. Y. \& Leung A. S. Corporate social responsibility, firm reputation, and firm performance : The role of ethical leadership. Asia Pacific Journal of Management, 2014,31(4), 925-947. 